\title{
Predictive Maintenance of Cash Dispenser Using a Cognitive Prioritization Model
}

\author{
Archana Dixit*, Amol B. Mahamuni
}

\begin{abstract}
In this technical paper, we address the issue of predicting cash dispenser (addressed as 'Device' henceforth) failure by harnessing the power of humungous data from service history, logs, metrics, transactions, and plausible environmental factors. This study helps increase device availability, enhanced customer experience, manage risk \& compliance and revenue growth. It also helps reduce maintenance cost, travel cost, labour cost, downtime, repair duration and increase meantime between failures (MTBF) of individual components. This study uses a cognitive prioritization model which entails the following at its core; a) Machine Learning engineered features with highest influence on machine failure, b) Observation Windows, Transition Windows and Prediction Windows to accommodate various business processes and service planning delivery windows, and c) A forward-looking evaluation of emerging patterns to determine failure prediction score that is prioritized by business impact, for a predefined time window in the future. The model not only predicts failure score for the devices to be serviced, but it also reduces the service miss impact for the prediction windows.
\end{abstract}

Keywords: Cash Dispenser Failure Prediction; Cognitive Prioritization Model; Feature Engineering; Machine Learning; Predictive Maintenance (PdM)

\section{INTRODUCTION}

Maintenance is a vital area that controls major cost savings and revenue. According to the International Society of Automation (ISA), more than $\$ 647$ billion is lost each year due to downtime. All businesses have strived to achieve cost effective maintenance, high availability and customer satisfaction. Retail Banks are no different. They too have grappled with maintenance processes to alleviate downtime for a long time. Lack of a formal commitment and the easiness with which consumers can switch to competitors make the process of building trust among consumers even tougher for retail bankers. The consumers need to be able to get the service they want on a desired time. Otherwise, $66 \%$ of them will simply switch banks on account of an unfulfilling service needs.

Cash dispenser devices are getting more and more powerful everyday by supporting capabilities such as Passbook updating, Cash-Cheque Deposits, Money Transfer, Statement printing, Voucher Printing, Electronic Purse Upload, Wire Transfer, Update Personal Information, CrossSelling, Advertising, Special Interest and so on. Sudden failure and unplanned maintenance of these devices can have substantial impact on not only banking specific services but also revenue generating streams. It also directly impacts customer relationships. Therefore, there is an urgent need to rise from preventative or reactive maintenance to predictive maintenance.

This study introduces predictive maintenance capabilities that can immensely help

- Identify failures in advance leading to the optimization of logistics and associated functions

- Lower downtime by a significant factor

- Spill over benefits from optimized cash in transit activities

- Increase reliability and operational efficiency.

This study uses three rolling time windows 'Observation', 'Transition' and 'Prediction' to determine explanatory features and window of prediction. Features pertaining to inventory and machine components are treated as static while features pertaining to maintenance/service history, errors generated by the machines, environmental changes, and transactions are treated as dynamic. Dynamic features are designed in view of aforementioned time windows. Observation window depicts the slice of data consumed by the model during model training and prediction generation. Transition window signifies the time required to process, prepare and make the data compatible to generate predictions. Predictions are generated for four weeks into the future to allow adequate time for engineering team to act upon the predictions and perform maintenance activities on the identified devices or their components.

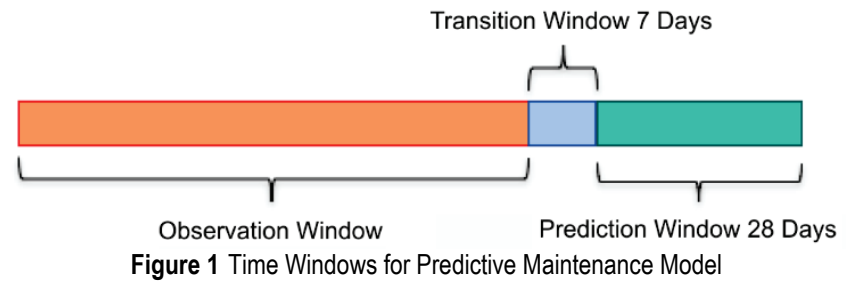

The remaining structure of this paper is organized as follows. Section 2 represents existing and related work on predictive maintenance. Section 3 focuses on the proposed method employed and describes the following; data used, exploratory data analysis, feature engineering, architecture of the proposed solution followed by model training and its evaluation. Section 4 discusses the results obtained in detail. Section 5 reviews the impact, stance and contribution of this solution. Section 6 highlights the most relevant aspects of this study and how it could easily be extended to other sectors. Finally, Section 7 concludes this paper by throwing light on the plausible approaches that could be leveraged in the future to make this Predictive maintenance solution more robust.

\section{RELATED WORK}

An extensive study of literature, purposes and available approaches is explained by the authors in [1]. Work carried out by other authors $[4,5]$ in this area also focuses primarily 
on generic approaches around predictive maintenance. Some of them focus on challenges and reliability aspect of PdM in their study [6], while others focus only on the cost and economic aspect $[2,15,19]$. While there are studies done for Industrial and IoT devices $[10,11,12,14,16]$, there are only a handful studies available for cash dispenser devices [21] wherein only event/error logs are considered as variables to perform Time Series Classification. Strong indicators of failures could be gleaned out from service history, metrics, transactions, etc. which is missing from the existing work in this field. The focus of this study is to harness the power of data points from service history, logs, metrics, transactions, and plausible environmental factors to get a holistic view around the device and component failure.

\section{PROPOSED METHOD}

This study uses a well-defined leading methodology used by industry data miners all over the world called Cross Industry Standard Process for Data Mining (CRISP-DM) (depicted in Fig. 2). It entails framing business questions, understanding data, preparing it for model training, modelling, evaluation and deployment.



Figure 2 Processes performed in CRISP-DM Methodology

This study leverages two years' worth of data with approx. 10K inventory. Multidimensional data encompasses crucial information from inventory, service history, error logs, transactions, and environment. The grain is at the device component level. Translation Activity Report (TAR Code) from inventory is used to identify modules/components for prediction (TAR Code structure is illustrated in Fig. 3). Preliminary analysis of data reveals that Cheque and Cash Deposit Module (CCDM) is the most profoundly used component of the device. Therefore, it is chosen to illustrate the approach used in this study.



\subsection{Exploratory Data Analysis (EDA)}

An extensive EDA is performed during this study to understand data distribution before applying any model. Univariate, Bivariate \& multivariate analysis is performed to understand distribution, association \& correlation of these variables better. The following Figs. 4, 5, $6 \& 7$ are some of the visuals (illustrative not exhaustive) depicting the same.

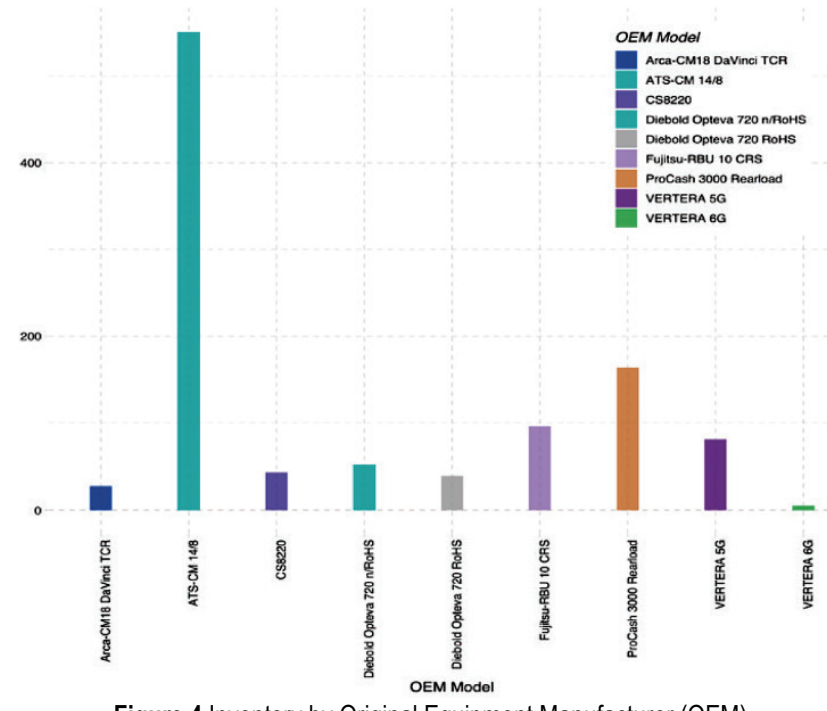

Figure 4 Inventory by Original Equipment Manufacturer (OEM)

Most of the inventory used in this study belongs to one specific OEM model. In addition, CCDM module accounts for most of the service tickets confirming that it is indeed the most often used component during transactions.

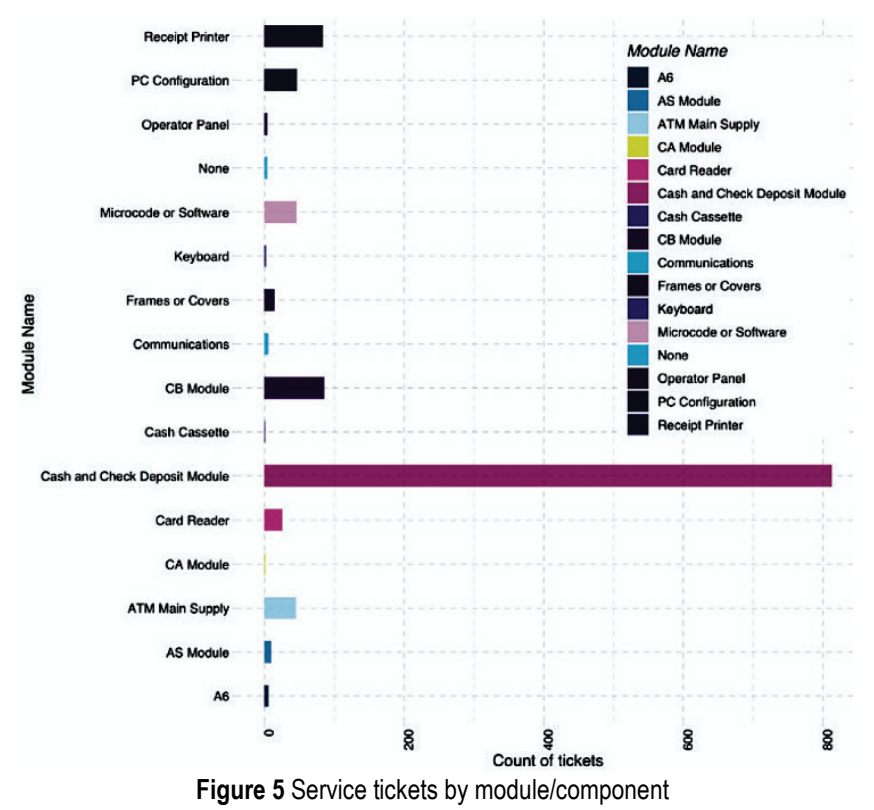

Service tickets show a downward trend, aptly so as banks strive hard to reduce these numbers (Fig. 6).

Fig. 7 depicts primary problem areas for the CCDM module. 

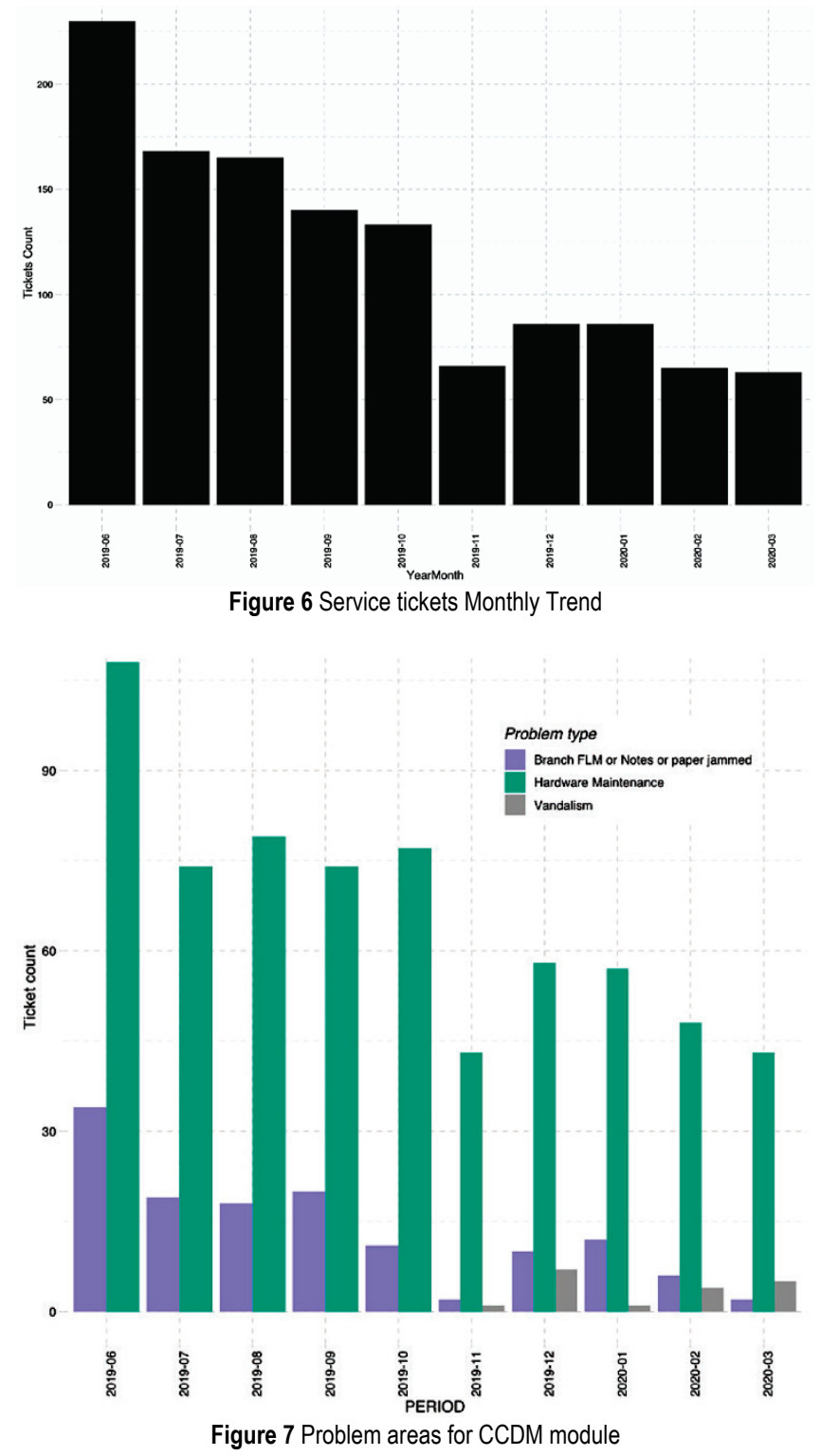

Our EDA does not exhibit any prominent issues with the data with respect to missing values or outliers. Data is mostly clean and tidy.

\subsection{Feature Engineering}

Predictions are generated at module or component level and therefore, features are also engineered for each module. As described earlier, there are two types of features used in this study; static \& dynamic. Static features are time windows independent and dynamic features respect time windows mentioned beforehand.

Inventory details like 'OEM', 'Age', 'TAR Action', 'TAR problem' etc. are static. Only active inventory (Devices that are not yet deinstalled) is considered for this study. 'Last Failure', 'Uptime', 'Last Visit' etc. are computed using Observation Window and most recently closed ticket. Transaction features 'Operation Count', 'Served Count', 'Reversed' etc. are created by performing aggregation. Error features 'Err Type 1 Count', 'Err Type 2 Count' and so on are computed for each error type. Error time elapsed features are also computed for each error type. Mean Time Between Failure (MTBF) features are computed too using error information. Features Avg MTBF, Weighted MTBF, etc. are computed using statistical measures. Ticket features 'Last Preventive', 'Preventive Ticket Count', 'Repair Count', etc. are computed using aggregation and business rules. These dynamic features contribute to significant chunk of explanatory variables used in this study. Target variable has two classes; 'Failure' \& 'Operational' making it a binary classification supervised machine-learning problem. All these features are generated for each module.

\subsection{Predictive Maintenance Architecture}

The following flow represents the predictive maintenance (PdM) architecture employed in this study (Fig. 8). Data from various sources is collected and integrated into a single coherent source. Various data wrangling and munging techniques are leveraged to make it tidy and compatible for machine learning model training. Features are generated with respect to both time windows and business rules.

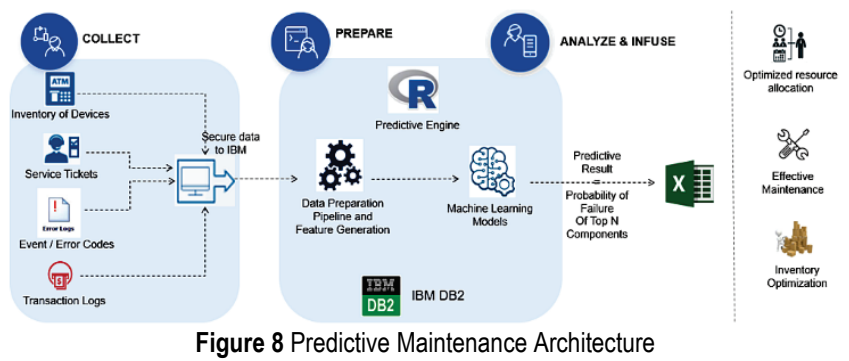

This study uses DB2 database to extract and store predicted results for subsequent actions. It leverages open source programming language $\mathrm{R}$ to perform data extraction, data preparation, data transformation, statistical modelling, machine learning model building, training \& evaluation. Meaningful actionable insights are also extracted using R's visualization libraries. Generated predictive insights are used for optimized resource allocation, effective maintenance and inventory optimization.

\subsection{MODEL TRAINING \& EVALUATION}

Data for CCDM module suffers from imbalance of classes; 'failure' \& 'Operational'. Therefore, sampling techniques; upsampling, downsampling, SMOTE are employed to create a dataset with balanced classes. Inbuilt weightage techniques provided in $\mathrm{R}$ libraries are also leveraged to overcome class imbalance issue. Cross validation and bootstrapping strategies are used in splitting train and test datasets to tackle overfitting issues. Numerous experiments are carried out for each module and algorithm combination by tuning various hyperparameter nobs to arrive at the optimal parameter values resulting in best performance 
of the model. Figs. 9 - 11 depict results for few of such experiments.

The following algorithms are used to perform model training and prediction of failure for each module; RandomForest, XGBoost, Support Vector Machines, Adaboost, and Generalized Linear Model. Multiple models are trained and validated before selecting a best one for scoring and prediction on new unseen data.

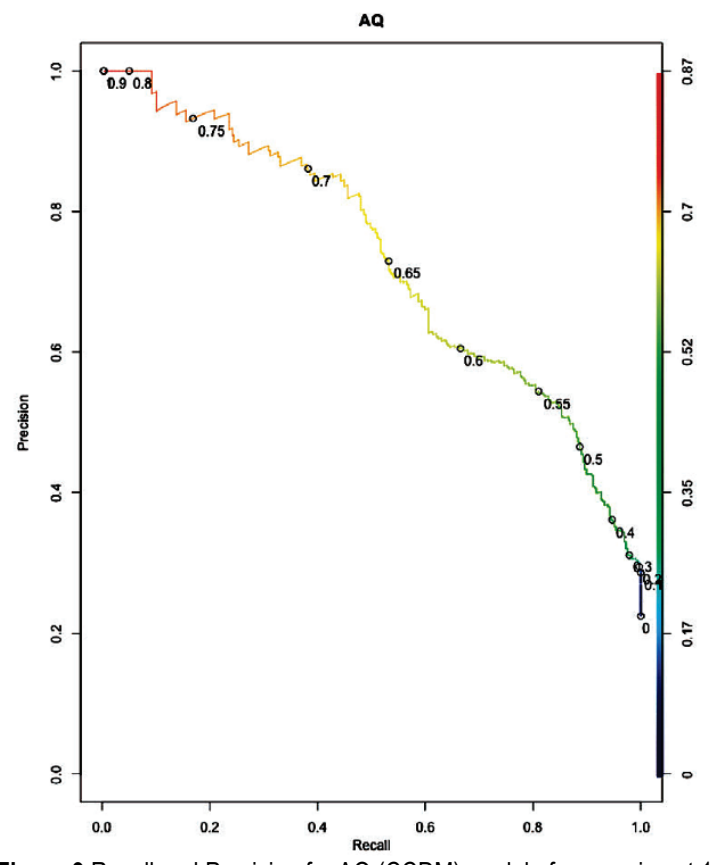

Figure 9 Recall and Precision for $A Q(C C D M)$ module for experiment 1

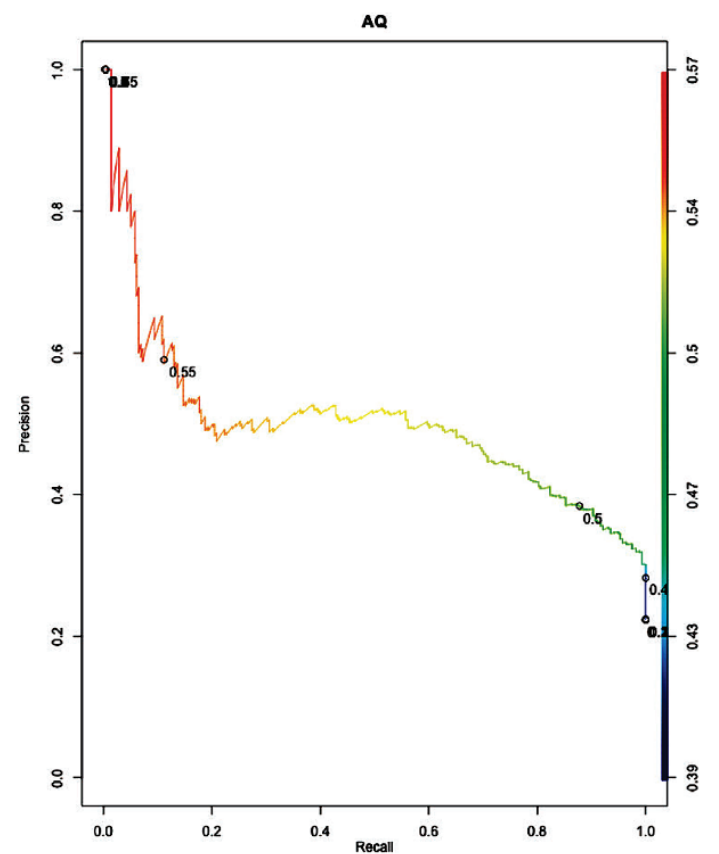

Figure 10 Recall and Precision for $A Q$ (CCDM) module for experiment 2

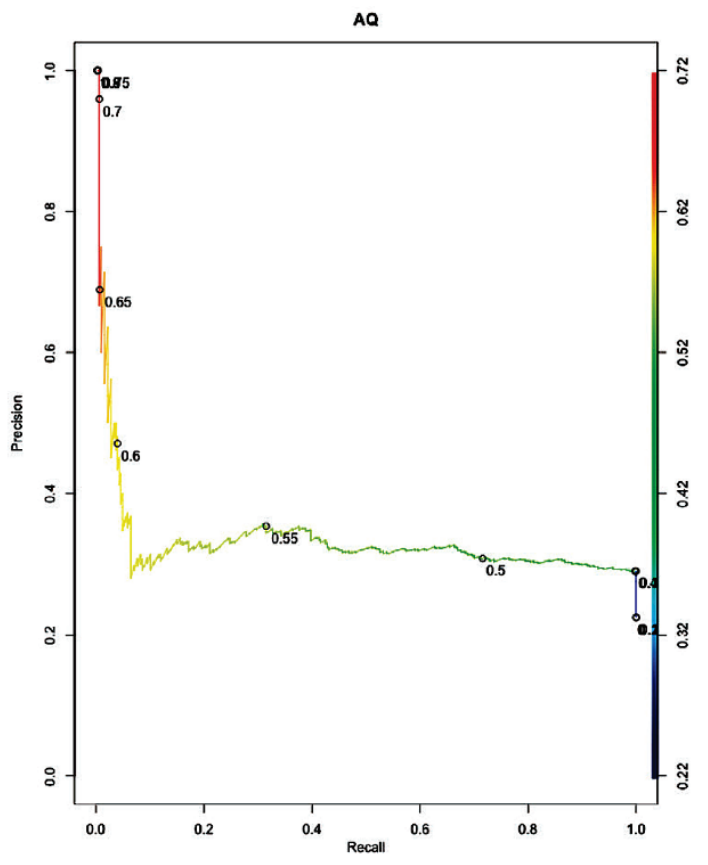

Figure 11 Recall and Precision for $A Q$ (CCDM) module for experiment 3

In this study we are more concerned about how many of the actual failures are predicted as failures by the model, i.e. recall rather than vanilla accuracy or the model. Recall is mathematically defined as the ratio of accurately classified positive classes by model and actual positive classes. Therefore, in our study we focused on reducing Type II error or false negatives to achieve the best possible recall.

\section{RESULTS}

Subsequently, it is observed that the ensemble algorithm Random Forest performs best for the data at hand. It gives us the highest recall and outperforms other algorithms. Generated insights could also be categorized as high, medium or low based on the prediction probability of each module/component to specify severity of the failure. High severity failures may then be attended to on a priority basis. This novel framework preserves time-driven business process in perspective while generating predictions to facilitate adaption based on relatedness, reliability and efficiency factors.



Figure 12 Recall and Precision for $A Q$ (CCDM) module using Random Forest 


\section{OUTLOOK \& IMPACT}

There may be a reduction of approx. $40-50 \%$ in reactive tickets if generated predictive insights are taken into account and acted upon well within the predictive window.

Fig. 13 describes predictions generated for two rounds for illustrative purposes. Prediction impact analysis is performed by visualizing device count with prediction failures vs device count with reactive tickets for failure in future prediction window. There were sixteen out of total thirty devices that were predicted in round 1 would have reactive tickets in future. Similarly, there were ten out of total twenty-five devices that were predicted in round 2 would have reactive tickets in future.

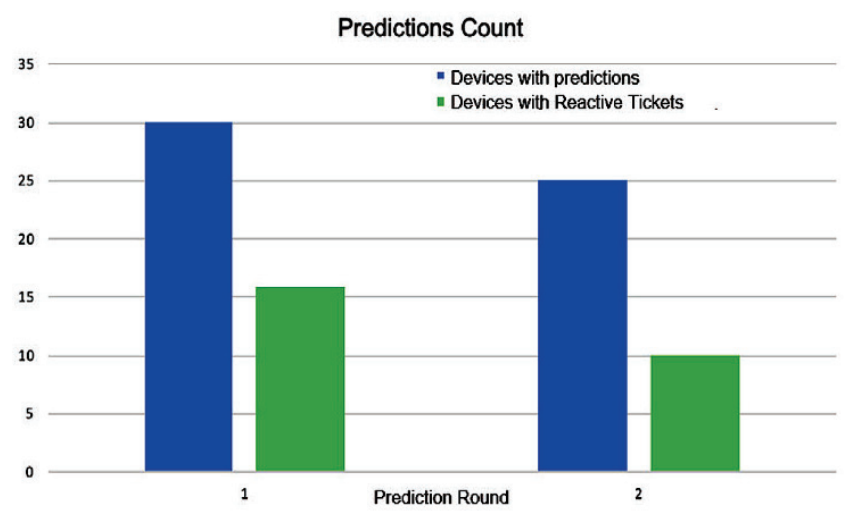

Figure 13 Predictions vs Reactive Tickets for Prediction Rounds

Further, such analysis shows that there is an increase in device availability by $2 \%$ if this solution is implemented for predictive maintenance. Meantime between failures of individual components is almost doubled. The solution identifies failures in advance leading to the optimization of logistics and associated functions. Downtime is also lowered by a significant factor. First touch resolution is increased within a week of ticket logging. Repair duration, labor cost and travel costs are lowered noticeably too. Retail banks are able to attain spillover benefits from optimized cash in transit activities, as they relate to second line maintenance.

\section{CONCLUSION}

In this work, we have studied how machine learning heuristic models augmented with sophisticated feature engineering mechanism could be used to empower retail bankers by predicting component that are mostly likely to fail in the next four weeks. Multidimensional data from disparate touch points of devices is employed to facilitate predictive maintenance in real time.

This study could easily be prolonged to predict failure in other devices such as POS machines, Self-checkout kiosks, etc.

\section{WAY FORWARD}

This study has several avenues of enhancement to add more capabilities into the overall Predictive maintenance solution.
- Sensor Data could be married with existing multidimensional data to enrich and form 360-degree view of devices and respective components

- Inclusion of data indicating any kind of social unrest leading to vandalism may help boost model's prediction power.

- This could easily be extended to predict failures in Data centers, servers, and other IT infrastructure devices

- The state-of-the-art techniques Deep Learning, LSTM, HTM may also be leveraged to improve model performance and thereby prediction capabilities

- Prescriptive capabilities could further enhance the solution and assist engineers in resolving issues with quick turnaround.

- This approach may be experimented with Python programming language to design an overall offering.

\section{Notice}

This paper was presented at IC2ST-2021 - International Conference on Convergence of Smart Technologies. This conference was organized in Pune, India by Aspire Research Foundation, January 9-10, 2021. The paper will not be published anywhere else.

\section{REFERENCES}

[1] Ran, Y., Zhou, X., Lin, P., Wen, Y., \& Deng, R. (2019). A Survey of Predictive Maintenance: Systems, Purposes and Approaches. Retrieved from: https://arxiv.org/abs/1912.07383

[2] S. Spiegel, F. Mueller, D. Weismann, J. Bird (2018). CostSensitive Learning for Predictive Maintenance. Retrieved from: https://arxiv.org/abs/1809.10979

[3] Tuggener, L., Amirian, M., Rombach, K., Lörwald, S., Varlet, A., Westermann, C., \& Stadelmann, T. (2019). Automated Machine Learning in Practice: State of the Art and Recent Results. https://doi.org/10.1109/SDS.2019.00-11

[4] Miller, K. \& Dubrawski, A. (2020). System-Level Predictive Maintenance: Review of Research Literature and Gap Analysis. Retrieved from: https://arxiv.org/pdf/2005.05239.pdf

[5] Bousdekis, A., Lepenioti, K., Apostolou, D., \& Mentzas, G. (2019). Decision Making in Predictive Maintenance: Literature Review and Research Agenda for Industry 4.0. IFACPapersOnLine, 52(13), 607-612. https://doi.org/10.1016/j.ifacol.2019.11.226

[6] Meyer Zu Wickern, V. - Challenges and Reliability of Predictive Maintenance. (2019). Challenges and Reliability of Predictive Maintenance. https://doi.org/10.13140/RG.2.2.35379.89129

[7] Poór, P. \& Basl, J. (2019). Predictive maintenance as an intelligent service in Industry 4.0. Journal of Systems Integration, 10(1), 3-10. https://doi.org/10.20470/jsi.v10i1.364

[8] Paolanti, M., Romeo, L., Felicetti, A., Mancini, A., Frontoni, E., \& Loncarski, J. (2018). Machine Learning approach for Predictive Maintenance in Industry 4.0. The $14^{\text {th }}$ IEEE/ASME International Conference on Mechatronic and Embedded Systems and Applications (MESA), 1-6. https://doi.org/10.1109/MESA.2018.8449150

[9] Schmidt, B., Sandberg, U., \& Wang, L. (2014). Next Generation Condition Based Predictive Maintenance. The $6^{\text {th }}$ International Swedish Production Symposium, 
https://www.researchgate.net/publication/318775487_Next_G eneration_Condition_Based_Predictive_Maintenance.

[10] Bonci, A., Longhi, S., \& Nabissi, G. (2019). Predictive Maintenance System using motor current signal analysis for Industrial Robot. The $24^{\text {th }}$ IEEE International Conference on Emerging Technologies and Factory Automation (ETFA), 1453-1456. https://doi.org/10.1109/ETFA.2019.8869067

[11] Cakir, M., Ali Guvenc, M., \& Mistikoglu, S. (2020). The Experimental Application of Popular Machine Learning Algorithms on Predictive Maintenance and the Design of IIoT based Condition Monitoring System. Computers \& Industrial Engineering, 106948. Available online 30 October 2020. https://doi.org/10.1016/j.cie.2020.106948

[12] Maashi, M., Alwhibi, N., Alamr, F., Alzahrani, R., Alhamid, A., \& Altawallah, N. (2020). Industrial Duct Fan Maintenance Predictive Approach Based on Random Forest. The $9^{\text {th }}$ International Conference on Information Technology Convergence and Services (ITCSE 2020), 177-184. https://doi.org/10.5121/csit.2020.100516

[13] Raza, A. \& Ulansky, V. (2016). Modelling of predictive maintenance for a periodically inspected system. Procedia CIRP 59, 95-101. https://doi.org/10.1016/j.procir.2016.09.032

[14] Vibha, K., Pal, H., Bendigeri, M. V., \& Panna, N. (2020). Development of Predictive Maintenance System of Motors. International Journal of Advanced Science and Technology, 29(7), 2344-2351.

[15] Jing Yu, D. T. \& Wing-Keung, A. L. (2020). An Economic Perspective on Predictive Maintenance of Filtration Units. Retrieved from: https://arxiv.org/abs/2008.11070

[16] Zheng, H., Paiva, A. R., \& Gurciullo, C. S. (2020). Advancing from Predictive Maintenance to Intelligent Maintenance with AI and IIoT. Retrieved from: https://aiotworkshop.github.io/ published/AIoT_4_Zheng_TechnicalPaper_KDD2020.pdf

[17] Serradilla, O., Zugasti, E., \& Zurutuza, U. (2020). Deep learning models for predictive maintenance: a survey, comparison, challenges and prospect. Retrieved form: https://arxiv.org/pdf/2010.03207.pdf

[18] Satta, R., Cavallari, S., Pomponi, E., Grasselli, D., Picheo, D., \& Annis, C. (2017). A dissimilarity-based approach to predictive maintenance with application to HVAC systems. Retrieved from:

https://arxiv.org/ftp/arxiv/papers/1701/1701.03633.pdf

[19] Alsyouf, I. (2007). The role of maintenance in improving companies' productivity and profitability. International Journal of Production Economics, 105(1), 70-78. https://doi.org/10.1016/j.ijpe.2004.06.057

[20] Guillaume, A., Vrain, C., \& Wael, E. (2020). Time series classification for predictive maintenance on event logs. Retrieved from: https://arxiv.org/pdf/1911.10996.pdf

\section{Authors' contacts:}

Archana Dixit, Data Scientist (Corresponding author) IBM India

archana.dixit@in.ibm.com

Amol B. Mahamuni, Program Director IBM India mamol@in.ibm.com 